ARTICLE

DOI: $10.1038 / s 41467-017-01248-2$

\title{
Conversion of invisible metal-organic frameworks to luminescent perovskite nanocrystals for confidential information encryption and decryption
}

\author{
Congyang Zhang ${ }^{1}$, Bo Wang ${ }^{1}$, Wanbin $\mathrm{Li}^{2}$, Shouqiang Huang${ }^{1}$, Long Kong ${ }^{1}$, Zhichun $\mathrm{Li}^{1}$ \& Liang $\mathrm{Li}^{1}$
}

Traditional smart fluorescent materials, which have been attracting increasing interest for security protection, are usually visible under either ambient or UV light, making them adverse to the potential application of confidential information protection. Herein, we report an approach to realize confidential information protection and storage based on the conversion of lead-based metal-organic frameworks (MOFs) to luminescent perovskite nanocrystals (NCs). Owing to the invisible and controlled printable characteristics of lead-based MOFs, confidential information can be recorded and encrypted by MOF patterns, which cannot be read through common decryption methods. Through our conversion strategy, highly luminescent perovskite NCs can be formed quickly and simply by using a halide salt trigger that reacts with the MOF, thus promoting effective information decryption. Finally, through polar solvents impregnation and halide salt conversion, the luminescence of the perovskite NCs can be quenched and recovered, leading to reversible on/off switching of the luminescence signal for multiple information encryption and decryption processes.

\footnotetext{
${ }^{1}$ School of Environmental Science and Engineering, Shanghai Jiao Tong University, 800 Dongchuan Road, 200240 Shanghai, People's Republic of China. ${ }^{2}$ School of Environment, Guangzhou Key Laboratory of Environmental Exposure and Health, and Guangdong Key Laboratory of Environmental Pollution and Health, Jinan University, 601 West Huangpu Road, 510632 Guangzhou, People's Republic of China. Correspondence and requests for materials should be addressed to L.L. (email: liangli117@sjtu.edu.cn)
} 
T he development of stimuli-responsive fluorescent materials has attracted particular attention due to their potential security protection applications such as information storage, encryption, and anti-counterfeiting ${ }^{1,2}$. With some external stimuli, the luminescent outputs of these materials can be tactfully changed, preventing the information or data from beingstolen, mimicked, or forged. In the past decades, a range of smart luminescent materials including transition-metal complexes ${ }^{3-5}$, organic dyes 6 , inorganic semiconductor nanocrystals $(\mathrm{NCs})^{7-9}$, carbon $\operatorname{dots}^{10-12}$, and lanthanide-doped upconverting nanoparticles $^{13-15}$ with changeable luminescent outputs have been explored. Through controlling their chemical constitutions or structures in the solid state, tunable luminescent signals can be achieved. These smart materials, however, suffer from many drawbacks such as insufficient luminescent performances, high material cost and/or tedious synthesis, and purification procedures. More importantly, due to their photoluminescent property, the data or informations recorded directly by these materials are usually visible under either ambient or UV light, which is adverse to their practical application for confidential information protection ${ }^{16}$. Therefore, it remains a great challenge to develop alternative cost-effective high-quality luminescent materials and systems with good confidential encryption abilities for high-level information storage and security protection applications.
Recently, metal halide perovskite $\mathrm{ABX}_{3} \quad(\mathrm{~A}=$ monovalent cations, $\mathrm{B}=$ divalent metal, and $\mathrm{X}=\mathrm{Cl}, \mathrm{Br}, \mathrm{I})$ materials have attracted great scientific interest in many research fields due to their excellent optoelectronic properties ${ }^{17-19}$. As fluorescent materials, low-cost lead halide perovskite nanomaterials exhibit bright photoluminescence (PL), relatively high PL quantum yields (PLQY), narrow emission spectra, and wide color gamut ${ }^{20-24}$. All the above advantages may make them excellent candidates for constructing smart luminescent systems ${ }^{25}$. However, direct use of perovskite nanomaterials as smart fluorescent systems is not a good choice because they are still visible under ambient or UV light, just like other fluorescent materials.

Herein, we propose an approach to realize confidential information protection and storage based on the conversion of leadbased metal-organic frameworks (MOFs) to luminescent perovskite NCs. As a relatively new class of porous hybrid materials, MOFs consist of inorganic metal centers and organic linkers by coordination bonds and possess a series of unique characteristics, including structure diversity, tunable pore sizes, tailorable functionality, and large surface area ${ }^{26-29}$. In our strategy, by employing MOFs as the lead source and the sacrificial porous template simultaneously, bright luminescent perovskite NCs in MOFs can be obtained via a direct conversion process simply triggered by a halide salt. Notably, the invisible and controlled a
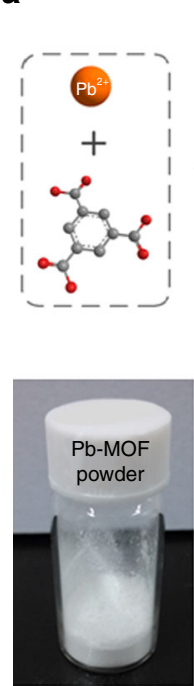

d

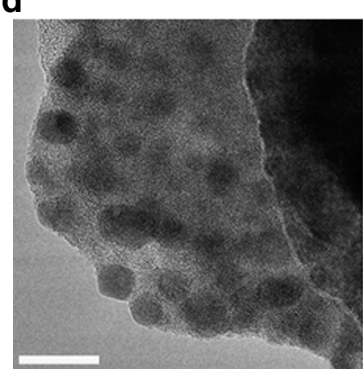

Pb-MOF
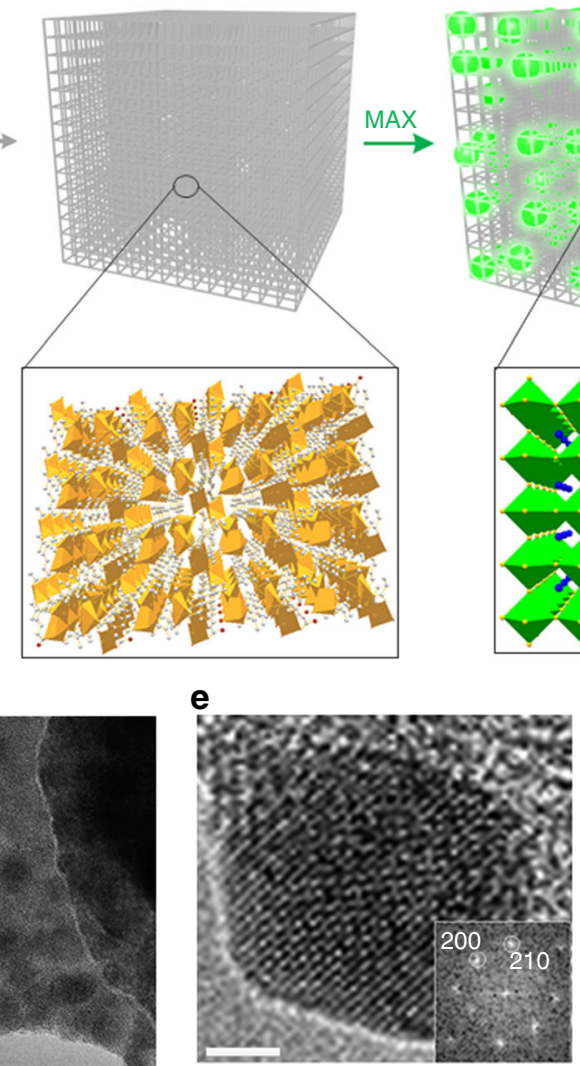

$\mathrm{MAPbBr}_{3} \mathrm{NCs} @ \mathrm{~Pb}-\mathrm{MOF}$

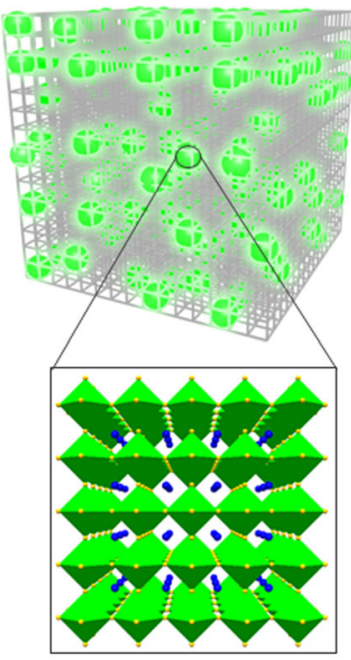

b

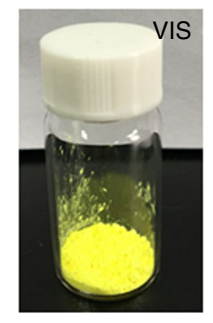

C

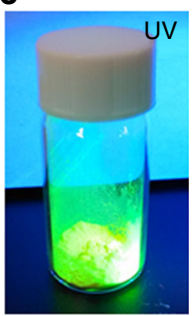

f

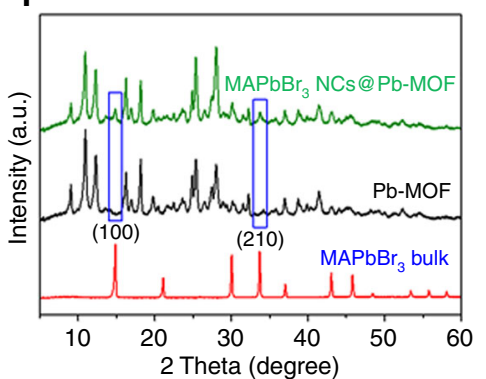

Fig. 1 Conversion of a Pb-MOF to luminescent $\mathrm{MAPbBr}_{3} \mathrm{NCs} @ \mathrm{~Pb}-\mathrm{MOF}$. a Schematic of the conversion process. MAX represents the halide salt $\left(\mathrm{CH}_{3} \mathrm{NH}_{3} \mathrm{X}, \mathrm{X}=\mathrm{Cl}, \mathrm{Br}\right.$, or I). The green spheres in the matrix represent the $\mathrm{MAPbBr}_{3} \mathrm{NCs}$. The two black boxes show 3D crystal structure of the Pb-MOF (left) and $\mathrm{MAPbBr}_{3}$ (right). The $\mathrm{Pb}$ coordination polyhedra of the $\mathrm{Pb}-\mathrm{MOF}$ (the $\mathrm{Pb}$ atom are coordinated by nine $\mathrm{O}$ atoms, in which two $\mathrm{O}$ atoms of one carboxylate coordinate to a $\mathrm{Pb}$ and also bridge two adjacent $\mathrm{Pb}$ atoms) and $\mathrm{MAPbBr}_{3}$ are represented in orange and green, respectively. Other atom color scheme: $\mathrm{C}=$ gray, $\mathrm{O}=$ red, $\mathrm{N}=$ blue, $\mathrm{Br}=$ yellow. $\mathrm{H}$-atoms have been omitted for clarity. $\mathbf{b}$, c Optical images of $\mathrm{MAPbBr}{ }_{3} \mathrm{NCs} @ \mathrm{~Pb}-\mathrm{MOF}$ powder under $\mathbf{b}$ ambient light and $\mathbf{c} 365 \mathrm{~nm}$ UV lamp; d-f Characterization of the MAPbBr 3 NCs@Pb-MOF: d TEM image, e HR-TEM image of one individual NC with the corresponding fast Fourier transformation image in the bottom right corner and $\mathbf{f}$ XRD patterns. Scale bar, $20 \mathrm{~nm}(\mathbf{d}) ; 5 \mathrm{~nm}(\mathbf{e})$ 

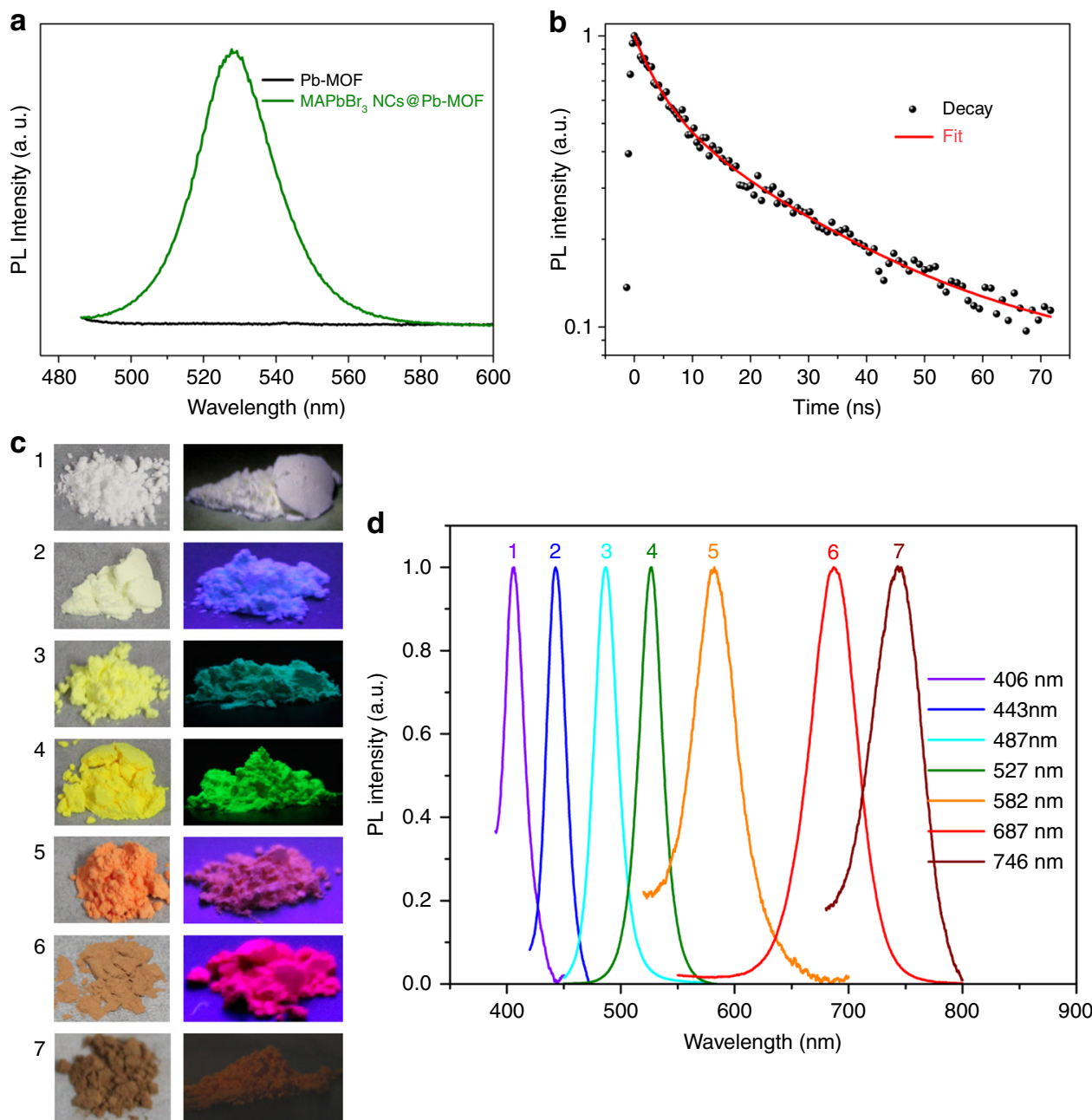

Fig. 2 Optical properties of $\mathrm{MAPbX}_{3} \mathrm{NCs@Pb-MOF.} \mathrm{a} \mathrm{Steady-state} \mathrm{PL} \mathrm{emission} \mathrm{spectra} \mathrm{of} \mathrm{Pb-MOF} \mathrm{(black} \mathrm{line)} \mathrm{and} \mathrm{MAPbBr} 3$ NCs@Pb-MOF (green line). b Time-resolved PL decay curve of $\mathrm{MAPbBr}_{3} \mathrm{NCs} @ \mathrm{~Pb}-\mathrm{MOF}$ detected at $527 \mathrm{~nm}$ with excitation of $450 \mathrm{~nm}$. c Optical images under ambient light and 365 nm UV lamp and $\mathbf{d}$ steady-state PL emission spectra of $\mathrm{MAPbX}_{3}$ NCs@Pb-MOF. (1: MAPbCl $, 2: \mathrm{MAPbCl}_{2} \mathrm{Br}, 3: \mathrm{MAPbClBr}_{2}, 4: \mathrm{MAPbBr}_{3}$, 5: MAPbBr $\mathrm{M}_{2}$, 6: $\mathrm{MAPbBr}_{2}, 7: \mathrm{MAPbl}_{3}$ )

printable characteristics of the lead-based MOFs allow us to easily record confidential information and protect them from general decryption methods (such as photochromic methods ${ }^{30}$ ). On the basis of the conversion strategy mentioned above, we successfully and easily realized the confidential information encryption and decryption process with various inkjet-printed patterns by using the invisible and stable $\mathrm{Pb}-\mathrm{MOF}$ precursor as a security ink. In addition, due to the inherent ionic structure, the perovskite NCs in MOF matrix can be destroyed by polar solvents impregnation, thus quenching the luminescence of the perovskite NCs and realizing the recovery or even reversible on/off switching of the luminescence signal for multiple information encryption and decryption cycles.

\section{Results}

Conversion process. Firstly, we demonstrated the feasibility of the conversion process and fabricated the $\mathrm{CH}_{3} \mathrm{NH}_{3} \mathrm{PbBr}_{3}$ $\left(\mathrm{MAPbBr}_{3}\right) \mathrm{NCs}$ from a lead-based MOF powder for a typical example. A lead-based $\mathrm{MOF}\left(\mathrm{Pb}_{2}(1,3,5-\mathrm{HBTC})_{2}\left(\mathrm{H}_{2} \mathrm{O}\right)_{4}, \mathrm{~Pb}-\right.$ MOF) was firstly synthesized according to a previous report (Fig. 1a) ${ }^{31}$. In this framework, $\mathrm{Pb}^{2+}$ is coordinated by $1,3,5-$ $\mathrm{HBTC}^{2-}$ and two $\mathrm{H}_{2} \mathrm{O}$ molecules with a square-pyramidal coordination geometry, resulting a $2 \mathrm{D}$ polymeric structure (Supplementary Fig. 1). Figure la schematically illustrates the formation process of the $\mathrm{MAPbBr}_{3} \mathrm{NCs}$ in $\mathrm{Pb}-\mathrm{MOF}$ matrix (named as $\mathrm{MAPbBr}_{3} \mathrm{NCs@Pb-MOF).} \mathrm{Different} \mathrm{from} \mathrm{the} \mathrm{con-}$ fined synthesis of pervoskite NCs in porous materials in recent reports $^{32-36}$, our strategy is based on a direct conversion process triggered by small amount of $n$-butanol solution containing $\mathrm{CH}_{3} \mathrm{NH}_{3} \mathrm{Br}$ (MABr). To better control the conversion process, we used hexane to disperse the MOF powder. The emission color of the suspension changed quickly from non-fluorescence to blue green then to yellow green (Supplementary Fig. 2), indicating an obvious quantum-confinement phenomenon due to the growth of the perovskite NCs. After several minutes, the bright powder (Fig. 1b) was collected by filtration, rinsed, and dried. As shown in Fig. 1c, the resulting $\mathrm{MAPbBr}_{3} \mathrm{NCs@Pb-MOF}$ powder shows brilliant green emissions under a UV lamp (365 nm).

Morphology and structure characterization. Figure $1 \mathrm{~d}$, e and Supplementary Fig. 3 show the transmission electron microscopy (TEM) and scanning electron microscopy (SEM) images of the $\mathrm{MAPbBr}_{3} \mathrm{NCs@Pb-MOF}$ sample, respectively. As shown in TEM image (Fig. 1d), the synthesized $\mathrm{MAPbBr}_{3} \mathrm{NCs}$ are well distributed in $\mathrm{Pb}-\mathrm{MOF}$ with a diameter of about $10-20 \mathrm{~nm}$. From the high-resolution transmission electron microscopy (HR-TEM) and the fast Fourier transformation (FFT) images (Fig. 1e), the interplanar distances of 2.98 and $2.62 \AA$, corresponding to the 
(200) and (210) crystal faces of the cubic $\mathrm{MAPbBr}_{3}$ crystal, respectively, can be easily confirmed ${ }^{21}, 37$. The SEM images (Supplementary Fig. 3) show that the surface of Pb-MOF crystals become rough after the conversion process. In addition, crystal structure was characterized by X-ray diffraction (XRD) and shown in Fig. 1f. The XRD pattern of Pb-MOF is similar to the literature $^{31}$. After conversion, the framework structure of $\mathrm{Pb}$ MOF is well retained. Apparently, we can preliminary confirm the existence of $\mathrm{MAPbBr}_{3} \mathrm{NCs}$ in $\mathrm{Pb}-\mathrm{MOF}$ from the two new peaks at $14.9^{\circ}$ and $33.7^{\circ}$ correspond to (100) and (210) planes of the cubic $\mathrm{MAPbBr}_{3}$ (space group: $\mathrm{Pm} 3 \mathrm{~m}$ No. 211). To further demonstrate the conversion process, on one hand, the $\mathrm{Pb}-\mathrm{MOF}$ and $\mathrm{MAPbBr}_{3} \mathrm{NCs@Pb-MOF}$ were analyzed by X-ray photoelectron spectroscopy (XPS). As shown in the full-range XPS spectra (Supplementary Fig. 4), the new signals of $\mathrm{Br}, \mathrm{N}$ species appear distinctly. For more detail analysis, compared to the $\mathrm{Pb}$ MOF, the $\mathrm{Pb} 4 f$ peaks shift to lower binding energies (BE) (from 139.0 to $138.7 \mathrm{eV}$ ) and slightly broaden for the $\mathrm{MAPbBr}_{3}$ $\mathrm{NCs@Pb-MOF}$ sample, suggesting the change of coordination chemistry of $\mathrm{Pb}$ atoms (the emerging $\mathrm{Pb}-\mathrm{Br}$ bond from $\mathrm{MAPbBr}_{3}$ $\mathrm{NCs})$. On the other hand, a long time $(48 \mathrm{~h})$ and high reaction concentration of $\mathrm{MABr}$ (20 times than used for luminescent $\mathrm{MAPbBr}_{3} \mathrm{NCs@Pb-MOF)}$ conversion process was conducted to consume almost all of the $\mathrm{Pb}$ from MOFs, which confirmed by the XRD characterization. As shown in Supplementary Fig. 5, the diffraction signals of $\mathrm{Pb}-\mathrm{MOF}$ framework have markedly reduced, whereas the sharp peaks of $\mathrm{MAPbBr}_{3}$ are shown predominately, which indicates that the $\mathrm{Pb}$ elements for $\mathrm{MAPbBr}_{3} \mathrm{NCs}$ are indeed from $\mathrm{Pb}-\mathrm{MOF}$ rather than the residual $\mathrm{Pb}^{2+}$ in $\mathrm{MOF}$ matrix. In general, all of the above results have robustly demonstrated the successful conversion process of $\mathrm{Pb}-\mathrm{MOF}$ to $\mathrm{MAPbBr}_{3}$ NCs. Furthermore, the percentage of the perovskite NCs in MOF matrix was estimated by XRF (only 3\%, Supplementary Table 1) and XPS analysis ( 19\%, Supplementary Table 2). Notably, the difference between these results (3\% vs. $19 \%)$ can be ascribed to the different detecting depths of the two analysis methods. Compared with XRF, the detecting depth of XPS is only several nanometer ${ }^{38,} 39$, which may suggest that perovskite NCs are mainly located in the outer part of MOF particle. Also by employing the MOFs as self-templates, a series of nanomaterials (such as metal sulfide: $\mathrm{CuS}^{40}, \mathrm{ZnS}^{41}$, and metal oxides: $\mathrm{ZnO}^{42}$ et al.) have been synthesized in previous reports. Accordingly, the formation mechanism of the $\mathrm{MAPbX}_{3} \mathrm{NCs}$ in $\mathrm{Pb}-\mathrm{MOF}$ in our work could be attributed to the synergetic roles of the dissolution rate of $\mathrm{Pb}^{2+}$ from the MOF, the diffusion rate of $\mathrm{MA}^{+}$and $\mathrm{X}^{-}$toward the $\mathrm{Pb}-\mathrm{MOF}$ and the formation rate of $\mathrm{MAPbX}_{3} \mathrm{NCs}$, in which the formation rate is larger than the dissolution and diffusion rates ${ }^{41}$.

Optical characterization. Figure 2a shows the PL spectra of assynthesized $\mathrm{MAPbBr}_{3}$ NCs@Pb-MOF and $\mathrm{Pb}-\mathrm{MOF}$ powder. Obviously, $\mathrm{Pb}$-MOF does not show any florescence signal in the visible range. But the $\mathrm{MAPbBr}_{3} \mathrm{NCs@Pb-MOF}$ exhibits a green emission peak at $527 \mathrm{~nm}$ with narrow $25 \mathrm{~nm}$ full-width-at-halfmaximum (FWHM). The relative sharp emission highlights the outstanding superiority of the luminescent perovskite NCs over the traditional smart fluorescence materials. The excitationemission matrix (EEM) spectrum of the $\mathrm{MAPbBr}_{3} \mathrm{NCs@Pb-}$ MOF powder, shown in Supplementary Fig. 6, reveals that the PL emission is not excitation wavelength dependent. The UV-vis absorption spectra (Supplementary Fig. 7) show that $\mathrm{MAPbBr}_{3}$ NCs@Pb-MOF exhibits a broad absorption at $350-550 \mathrm{~nm}$. In contrast, the $\mathrm{Pb}-\mathrm{MOF}$ also does not show any absorption signal in the visible range, thus further indicating the "invisible" characteristic of the Pb-MOF. "Invisible" is in quotation marks because the Pb-MOF powder here, showing white color (Fig. 1b), is still visible under ambient light due to the existing scattering phenomenon. We should note that the PL peak or excitonic absorption peak wavelength of our bright powder sample is larger than the reported $\mathrm{MAPbBr}_{3}$ quantum dots (QDs) ${ }^{21,35}$, which can be attributed to the large crystal size compared to the small excitonic Bohr radius for $\mathrm{MAPbBr}_{3}(\sim 1.4-2 \mathrm{~nm})^{43}$. We hold that it is the final rinsing or drying step rather than conversion process that leading to the relative large crystal size of our perovskite sample because the quantum-confinement phenomenon can be obviously observed during the perovskite NCs' growth process (Supplementary Figs. 2 and 8). In addition, the absolute PLQY of the as-synthesis $\mathrm{MAPbBr}_{3}$ NCs@Pb-MOF powder is $39.6 \%$ determined by a fluorescence spectrometer with an integrated sphere executed at a wavelength of $390 \mathrm{~nm}$. Compared to the luminescent perovskite NCs synthesized by conventional solution-processable strategies initially outlined by Pérez-Prieto et al. ${ }^{20}$, the relatively lower PLQYs of the $\mathrm{MAPbBr}_{3} \mathrm{NCs@Pb-}$ MOF can be attributed to the absence of any surface shelling and insufficient ligand passivation. In spite of this, it is comparable to or even brighter than these reported $\mathrm{MAPbBr}_{3} \mathrm{NCs}$ confined synthesized in porous matrix ${ }^{32-36}$, and sufficient for information identification applications. The time-resolved PL spectrum is shown in Fig. 2b. The PL decay can be described by biexponential fitting, giving a short-lived PL lifetime $\left(\tau_{1}\right)$ of 4.4 ns with a percentage of $44.3 \%$ and long-lived PL lifetime $\left(\tau_{2}\right)$ of $26.2 \mathrm{~ns}$ with a percentage of $55.7 \%$. Similar to the previous report ${ }^{35}$, the shorter lifetime could be the result of dominant surface trapping of the $\mathrm{MAPbBr}_{3} \mathrm{NCs}$, suggesting that the non-radiative recombination pathway has non-negligible contribution in our $\mathrm{MAPbBr}_{3}$ NCs@Pb-MOF sample, which is consistent with the abovementioned result of relative low PLQY. Moreover, we have demonstrated that the conversion process also can be applied to the $\mathrm{CsPbX}_{3} \mathrm{NCs}$. As a representative, the $\mathrm{CsPbBr}{ }_{3} \mathrm{NCs}$ has been fabricated via a similar conversion process from the $\mathrm{Pb}-\mathrm{MOF}$. The optical properties and photograph of $\mathrm{CsPbBr}_{3} \mathrm{NCs} @ \mathrm{~Pb}$ MOF are shown in Supplementary Fig. 9, which indicates the versatility of our conversion approach. In addition, by adjusting their halide composition $(\mathrm{X}=\mathrm{Cl}, \mathrm{Br}$, and $\mathrm{I})$, the emission color of as-synthesized $\mathrm{MAPbX}_{3} \mathrm{NCs} @ \mathrm{~Pb}-\mathrm{MOF}$ can be tuned over the entire visible spectral region. Figure $2 \mathrm{c}$ and $\mathrm{d}$ show the optical images (under ambient and UV lamp) and the PL emission spectra of a series of $\mathrm{MAPbX}_{3}$ NCs@Pb-MOF samples with varied halide compositions, which has been easily tuned from deep blue to near infrared with relative narrow emissions $(\mathrm{FWHM}=19-55 \mathrm{~nm}$ ). From the XRD characterization (Supplementary Fig. 10), the peaks of the (100) and (210) reflection gradually shift toward higher angles with smaller halide ions $(\mathrm{Br}$, $\mathrm{Cl}$ ) due to the reduced lattice parameters, which confirm the cubic perovskite phase for all $\mathrm{MAPbX}_{3} \mathrm{NCs}$ samples.

Confidential information encryption and decryption application. Benefiting from the above "invisible" advantage of the $\mathrm{Pb}-\mathrm{MOF}$ and the robust conversion strategy of luminescent perovskite NCs, our perovskite NCs-MOFs platform may has great potential to realize the confidential information encryption and decryption process. Moreover, inspired by previous reports, the precise control of positioning and patterning of MOFs with high-resolution can be easily realized ${ }^{44-46}$, which offers another advantage of our platform for large area and high-density printable applications ${ }^{47}, 48$. Among numerous patterning technologies, inkjet printing is particularly attractive because of the maskfree, high-spatial resolution, and continuous operation advantages $^{49-51}$. In this manuscript, an invisible and stable precursor solution of $\mathrm{Pb}-\mathrm{MOF}$ has been used as the security ink directly to 

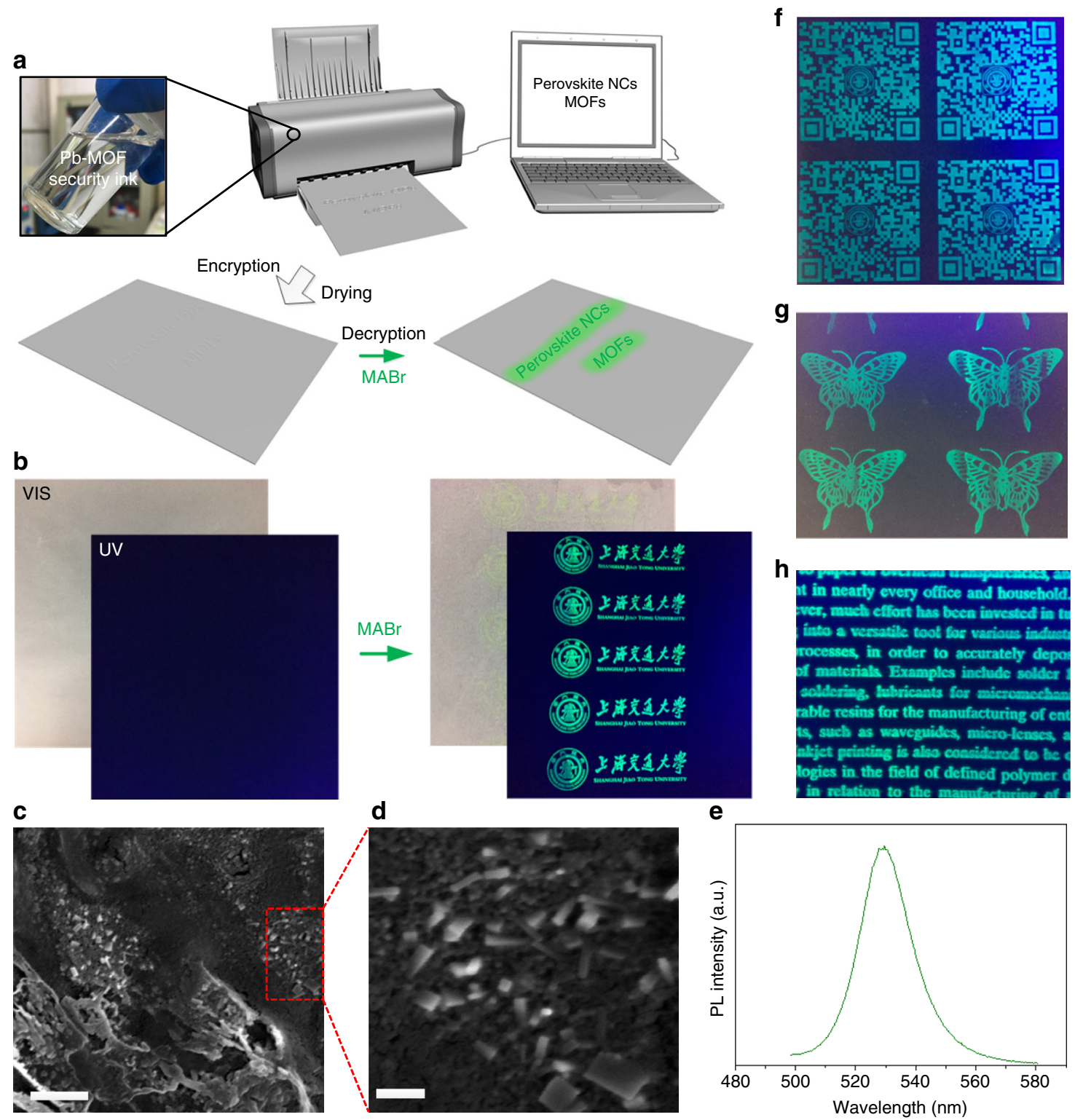

Fig. 3 Luminescent perovskite NC patterns from a Pb-MOF via inkjet printing. a Schematic illustrations of the patterning, information encryption, and decryption process of the perovskite NCs-MOF platform. b Digital images of the printed logo of Shanghai Jiao Tong University on a commercial parchment paper before and after MABr loading under ambient light and a $365 \mathrm{~nm}$ UV lamp. c, d SEM images of the parchment substrates with Pb-MOF pattern. Scale bar, $1 \mu \mathrm{m}$ (c); $200 \mathrm{~nm}$ (d). e PL spectrum of the MAPbBr 3 NCs@Pb-MOF pattern on parchment substrate. f-h Printed complicated patterns: QR code, butterfly, and characters, respectively

print various patterns by an inkjet printer. It is worth mentioning that the viscosity and surface tension of the ink have important role for the inkjet-printing process. Therefore, inspired by Zhuang's report ${ }^{52}$, a combinational solvent system containing dimethylsulfoxide (DMSO), ethanol, and ethylene glycol (EG) has been employed to prepare the $\mathrm{Pb}-\mathrm{MOF}$ precursor solution. Figure $3 \mathrm{a}$ illustrates the patterning, information encryption, and decryption process of our perovskite NCs-MOF platform. Through the inkjet-printing process, MOF precursor can be easily deposited onto desired positions using a nozzle. After the solvent evaporated by the drying step, small MOF crystals would nucleate and grow in specific areas. Notably, the printed Pb-MOF nanoscale crystals and the "invisible" characteristic of the $\mathrm{Pb}$ MOF jointly promote the absolutely and really invisible characteristic of $\mathrm{Pb}-\mathrm{MOF}$ patterns because of the reduced or even negligible scattering ${ }^{53-55}$, which is significant and necessary for confidential information encryption. Then the information decryption process can be conducted by conversion reaction of $\mathrm{Pb}$-MOF crystal on substrate via loading $n$-butanol solution containing MABr by a sprayer. About several minutes later, the solvent has been evaporated and the bright green emission pattern appeared clearly under UV light excitation.

Figure $3 \mathrm{~b}$ illustrates the printed logo of Shanghai Jiao Tong University on a commercial parchment paper. Obviously, the printed $\mathrm{Pb}-\mathrm{MOF}$ pattern is indeed invisible absolutely. The XRD (Supplementary Fig. 11) shows the crystalline characteristics of the above-mentioned $\mathrm{Pb}-\mathrm{MOF}$ with a partial preferential orientation mainly in the (001), (100), (101) directions. The SEM images of the printed Pb-MOF pattern, shown in Fig. $3 \mathrm{c}$ and $\mathrm{d}$, suggests that the $\mathrm{Pb}-\mathrm{MOF}$ pattern is composed of many nanoscale crystals (about $200-400 \mathrm{~nm}$ ). For the security protection applications, the stability of MOF seems to be a critical factor. As shown in Supplementary Fig. 12a, the thermogravimetry analysis (TGA) shows that the as-synthesized $\mathrm{Pb}-\mathrm{MOF}$ is 
a

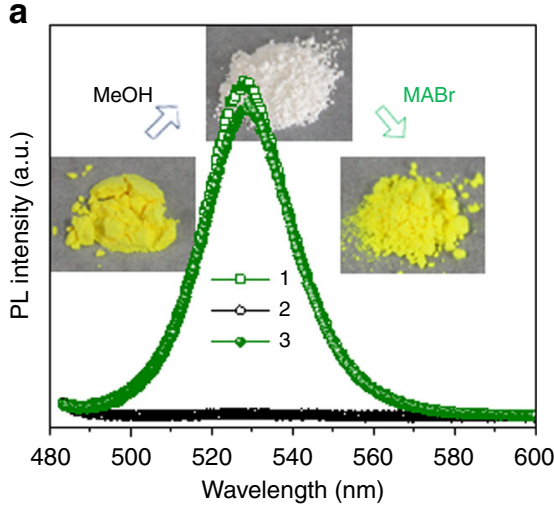

b

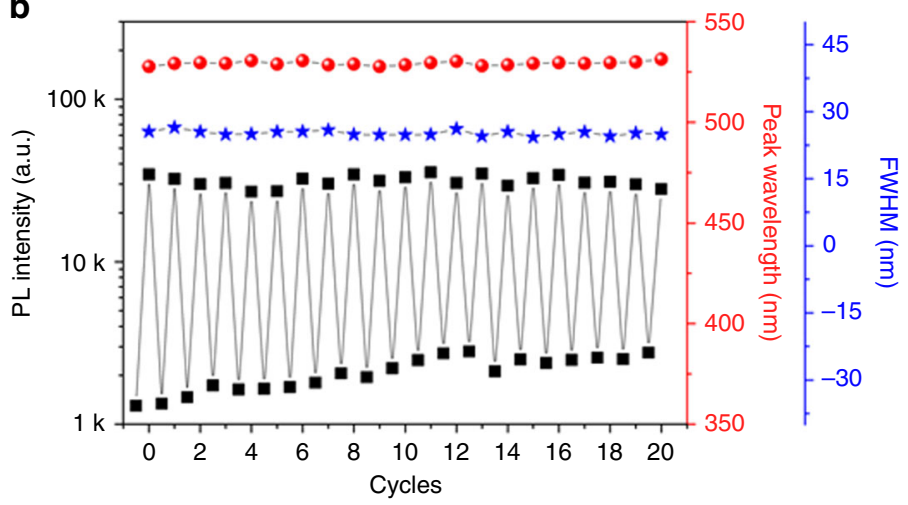

C
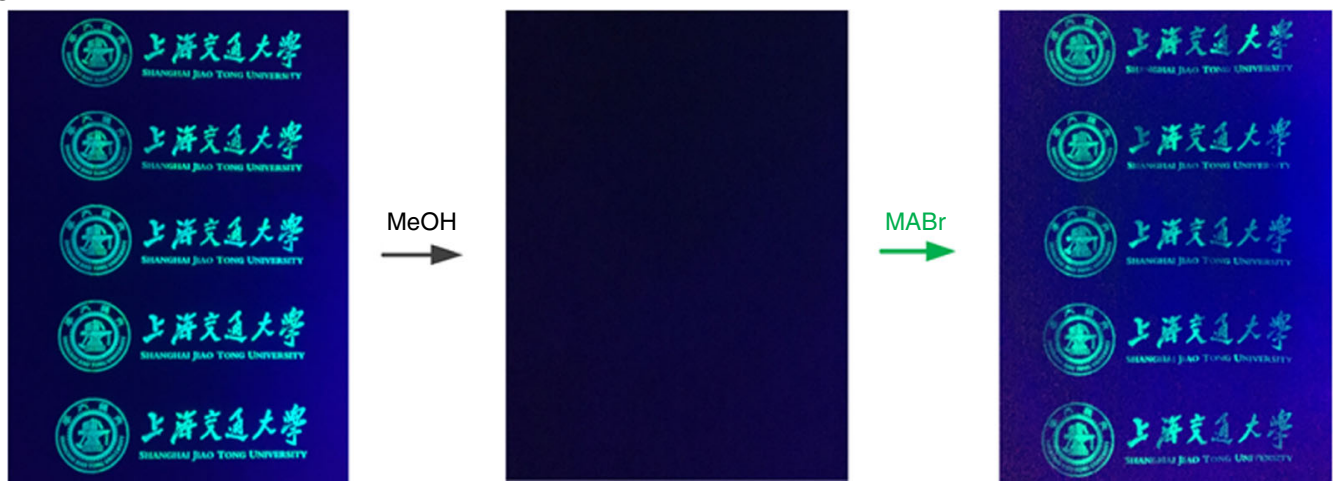

Fig. 4 The reversible on/off switching property of perovskite NCs-MOF platform. a Sequential optical images and PL emission spectra of MAPbBr 3 NCs@Pb-MOF after one cycles of impregnation-recovery process. 1, 2, and 3 represent the original, impregnated, and recovered powder sample of $\mathrm{MAPbBr}_{3} \mathrm{NCs} @ \mathrm{~Pb}-\mathrm{MOF}$, respectively. b PL intensity, peak wavelength, and FWHM of $\mathrm{MAPbBr}_{3} \mathrm{NCs} @ \mathrm{~Pb}-\mathrm{MOF}$ in the impregnation-recovery cycles as a function of cycle number. c Reversible fluorescence switching of the $\mathrm{MAPbBr}_{3} \mathrm{NCs} @ \mathrm{~Pb}-\mathrm{MOF}$ pattern in one encryption-decryption cycle (methanol impregnation for encryption and $\mathrm{MABr}$ spraying for decryption)

stable to $400{ }^{\circ} \mathrm{C}$ indicating that it has a good thermal stability, which is consistent with previous report ${ }^{31}$. On the other hand, from the XRD characterization (Supplementary Fig. 12b, c), it is obviously that both the $\mathrm{Pb}-\mathrm{MOF}$ powder and the printed $\mathrm{Pb}$ MOF pattern can remain original crystal structure after storage of several months, thus suggesting the excellent storage stability. After loading of $\mathrm{MABr}$, the logo comes out very pale yellow green color under ambient light and bright green color under UV light illumination. To be sure, benefiting from the excellent fluorescent properties of the perovskite NCs (high PLQY, sharp emission), the almost colorless and invisible $\mathrm{MAPbBr}_{3} \mathrm{NCs@Pb-MOF}$ pattern under ambient light also could be obtained by using MOF precursor with extremely low concentration without affecting the decryption process. The PL spectrum (Fig. 3e) reveals that the MAPbBr 3 NCs@Pb-MOF pattern on parchment paper has a narrow emission peak at $529 \mathrm{~nm}$, similar to the above bright powder sample. From the XRD characterization of the printed $\mathrm{MAPbBr}_{3} \mathrm{NCs@Pb-MOF}$ pattern in Supplementary Fig. 11, three typical diffraction peaks of cubic $\mathrm{MAPbBr}_{3}$ appear obviously. Moreover, as shown in Fig. $3 \mathrm{f}-\mathrm{h}$, various complicated patterns (including QR code, butterfly, and characters) have also been printed with good resolution. To demonstrate the necessity and the role of the MOF structure in the security protection application of our platform, the corresponding $\mathrm{Pb}^{2+}$ ink (without $\mathrm{H}_{3}$ BTC linker) was prepared and used for information encryption and decryption process. As shown in Supplementary Fig. 13, it is obvious that the printed pattern using $\mathrm{Pb}^{2+}$ ink cannot maintain the information encryption and decryption capability on substrates. The mechanical properties of the $\mathrm{Pb}-\mathrm{MOF}$ and $\mathrm{MAPbBr}_{3} \mathrm{NCs@Pb-MOF}$ pattern were also qualitatively assessed through a typical tape peel test $\mathrm{t}^{56,57}$. As shown in Supplementary Fig. 14, after tape adhesion and peeling, the $\mathrm{Pb}-\mathrm{MOF}$ and $\mathrm{MAPbBr}_{3}$ NCs@Pb-MOF pattern both can kept their high quality, which indicates that printed $\mathrm{Pb}-\mathrm{MOF}$ and $\mathrm{MAPbBr}_{3}$ NCs@Pb-MOF materials have excellent mechanical stability for security protection applications. Importantly, we also find that the obtained $\mathrm{MAPbBr}_{3}$ NCs@Pb-MOF pattern on parchment paper also exhibits good stability stored in air. As shown in Supplementary Fig. 15, the $\mathrm{MAPbBr}_{3}$ NCs@Pb-MOF pattern exposed in air after 3 months is invisible under ambient light but still exhibits green emission under the UV illumination. This phenomenon can be ascribed to the good protection effect of the textured substrate and the MOF matrix ${ }^{36}$.

Universality of perovskite NCs-MOF platform. Apart from parchment paper, the patterns also can be printed on transparent PET foils as well (Supplementary Fig. 16), enabling our perovskite NCs-MOF system's great potential for promising applications on multi-integrated light sources or other optoelectronic devices $^{58,59}$. In addition, the unique color tunable property of luminescent perovskite materials allow us to change the emission color of our patterns via replacing $\mathrm{MABr}$ with other halide salt. Supplementary Figure 17 illustrates the deep red colored patterns using an iodine-containing chromogenic reagent. Because of the relatively lower PLQY of the iodine-containing perovskite NCs, the deep red colored pattern is also a little bit dull. To further present the versatility of this strategy, as shown in Supplementary Fig. 18, we printed the letters of SJTU (the acronym of Shanghai Jiao Tong University) on parchment paper via contactprinting technique $e^{60}$. For more practical application, our 
perovskite NCs-MOF platform also shows good performance for anti-counterfeiting application on banknotes (Supplementary Fig. 19).

Reversible on/off switching of luminescence signals. Owing to the inherent ionic structure, the pervoskite framework is vulnerable and can be destroyed easily by many harsh conditions ${ }^{61-65}$. Inspired by this unique property, herein, we find that the $\mathrm{MAPbBr}_{3} \mathrm{NCs}$ in $\mathrm{Pb}-\mathrm{MOF}$ can be destroyed by polar solvents impregnation (e.g., methanol), enabling the quenching of the luminescence of perovskite NCs and may realizing the reversible on/off switch of the luminescence signal for multiple information encryption and decryption processes. Figure 4a displays the photographs and the PL emission spectra of the $\mathrm{MAPbBr}_{3}$ $\mathrm{NCs@Pb-MOF}$ powder samples within one cycle of impregnation-recovery process. After methanol impregnation and reaction with MABr again, the color of the as-synthesized powder changes to white and backs to yellow green. The assynthesized $\mathrm{MAPbBr}_{3} \mathrm{NCs} @ \mathrm{~Pb}-\mathrm{MOF}$ powder exhibits a strong fluorescence peak centered at $528 \mathrm{~nm}$ (curve 1). After impregnation and rinsing with methanol, the fluorescence of the sample is markedly quenched: the PL intensity decreases to only $0.4 \%$ (curve 2). Further, when the discolored powder reacted with $\mathrm{MABr}$ again, the fluorescence recovered and the PL intensity reaches $93.8 \%$ of the original value (curve 3 ). From the XRD and Fourier transform infrared (FTIR) data (Supplementary Fig. 20a, b) of the $\mathrm{MAPbBr}_{3} \mathrm{NCs@Pb-MOF}$ powder sample after methanol impregnation, the distinct diffraction peak at $14.9^{\circ}$ and the weak absorption peak at $977 \mathrm{~cm}^{-166}$ both disappear quickly. Furthermore, the XPS characterization of the $\mathrm{MAPbBr}_{3} \mathrm{NCs@Pb-MOF}$ powder, shown in Supplementary Fig. 20c and d, clearly reveals that after degradation, the signal of $\mathrm{Br}$ significantly reduced, whereas the $\mathrm{N}$ species almost disappeared. All of these data suggest that the luminescent $\mathrm{MAPbBr}_{3}$ phase can been degraded by the methanol treatment. Considering the small amount of the perovskite NCs and the complex hybrid system, it is difficult to determine the accurate degradation products from these results. To further figure out the degradation pathway, the methanol impregnation experiment of $\mathrm{MAPbBr}_{3}$ bulk sample has been directly conducted to avoid the influence of the MOF matrix (the $\mathrm{Pb}-\mathrm{MOF}$ does not change or degrade in methanol). The results (including XRD and FTIR), shown in Supplementary Fig. 21, demonstrate that after methanol impregnation, the $\mathrm{MAPbBr}_{3}$ NCs may mainly decompose into $\mathrm{PbBr}_{2}$ and other C, Ncontaining organic constituents, which can be easily washed away because of their good solubility (in methanol) and volatility. However, based on the small amount of $\mathrm{MAPbBr}_{3} \mathrm{NCs}$ in $\mathrm{Pb}-$ $\mathrm{MOF}$, the $\mathrm{Pb}$ element from the destroyed $\mathrm{MAPbBr}_{3} \mathrm{NCs}$ may have a relatively small role for the next conversion process. In this regard, the $\mathrm{Pb}-\mathrm{MOF}$ actually acts as a huge reservoir of metal source for the repeated formation of luminescent perovskite NCs, which allows the reversible on/off switching with high-quality fluorescent property of our platform. To further examine the reversible property, 20 cycles were conducted. As shown in Fig. 4 b, negligible decrease in PL intensity is observed after 20 consecutive switching cycles. In addition, the peak wavelength and FWHM of the PL spectra almost remain the same. Similar to the powder, the luminescence of $\mathrm{MAPbBr}_{3} \mathrm{NCs@Pb-MOF}$ pattern can also be quickly quenched by the methanol impregnation, and recovered again by $\mathrm{MABr}$ loading with high quality (Fig. 4c). Moreover, even after 10 consecutive switching cycles, the quality of the information encryption and decryption process still remains unaffected (Supplementary Fig. 22), which indicates that our platform can be applied for multiple information encryption and decryption processes.

\section{Discussion}

In summary, we have developed an approach to realize confidential information protection based on the conversion of invisible $\mathrm{Pb}$-based $\mathrm{MOFs}$ to luminescent perovskite NCs. Through simply reacting with $\mathrm{CH}_{3} \mathrm{NH}_{3} \mathrm{X}(\mathrm{MAX}, \mathrm{X}=\mathrm{Cl}, \mathrm{Br}$, or I) salt, the $\mathrm{MAPbX}_{3} \mathrm{NCs}$ with bright luminescence can be rapidly obtained in a Pb-MOF matrix. Owing to the invisible and controlled printable characteristic of the $\mathrm{Pb}-\mathrm{MOF}$ and the conversion process of luminescent perovskite NCs, we have demonstrated that our platform can act as a smart luminescent system towards confidential information encryption and decryption with various high-resolution patterns by inkjet-printing technique, which protect the recorded confidential information from general decryption methods. In addition, the inherent ionic structure of the perovskite materials allows us to quench the luminescence of the as-synthesized perovskite NCs easily by polar solvent (methanol) impregnation and realize the reversible on/off switching of the luminescence signal for multiple information encryption and decryption processes. It should be mentioned that the toxicity of $\mathrm{Pb}$ in lead halide perovskites ${ }^{67-69}$ is a considerable concern for the future applications of our platform. However, it would not be a fatal problem according to the recent fascinating research progresses in the synthetic chemistry of lead-free perovskite materials. Jellicoe et al. ${ }^{70}$ and Wang et al. ${ }^{71}$ have both reported successful replacement of lead with non-toxic tin by synthesizing $\mathrm{CsSnX}_{3}$ and $\mathrm{Cs}_{2} \mathrm{SnI}_{6} \mathrm{NCs}$, respectively. In addition, Leng et al. ${ }^{72}$ prepared a novel $\mathrm{MA}_{3} \mathrm{Bi}_{2} \mathrm{Br}_{9}$ QDs with high PLQY, suggesting that bismuth is another promising choice for toxic metal-free perovskite materials. We believe that our strategy will open up a potential avenue for luminescent perovskite materials in security-protecting applications.

\section{Methods}

Materials. Lead nitrate $\left(\mathrm{Pb}\left(\mathrm{NO}_{3}\right)_{2}, 99 \%\right)$, trimesic acid $\left(1,3,5-\mathrm{H}_{3} \mathrm{BTC}, 98 \%\right)$, methylamine $\left(\mathrm{CH}_{3} \mathrm{NH}_{2}, 30-33 \mathrm{wt}\right.$ \% in methanol solution), hydrobromic acid ( $\mathrm{HBr}, 48 \%$ in water), hydroiodic acid (HI, $57 \%$ in water), cesium bromide (CsBr, 99.9\%), cesium iodide (CsI, 99.9\%), dimethylsulfoxide (DMSO, >99\%) were acquired from Aladdin. Butanol $(\geq 99.5 \%)$, methanol ( $\geq 99.5 \%)$, $n$-hexane $(\geq 97 \%)$, toluene $(\geq 99.5 \%)$, ethanol ( $\geq 99.7 \%)$, ethylene glycol (EG, $\geq 99 \%)$, acetic acid ( $\mathrm{HCl}$, $\geq 99.5 \%$ ) were purchased from Sinopharm Chemical Reagent Co. Ltd, China.

Synthesis of Pb-MOF. The Pb-MOF $\left(\left[\mathrm{Pb}_{2}\left(1,3,5-\mathrm{H}_{3} \mathrm{BTC}\right)_{2}\left(\mathrm{H}_{2} \mathrm{O}\right)_{4}\right] \cdot \mathrm{H}_{2} \mathrm{O}\right)$ was synthesized following the previous procedure reported by Sadeghzadeh with some modification $^{31}$. Briefly, $90 \mathrm{~mL}$ of a $0.01 \mathrm{M}$ solution of $1,3,5-\mathrm{H}_{3}$ BTC in water was positioned in a high-density ultrasonic cleaner and $10 \mathrm{~mL}$ of a $0.09 \mathrm{M} \mathrm{Pb}\left(\mathrm{NO}_{3}\right)_{2}$ in water was added dropwise to the solution at room temperature. After addition, the solution remained in the bath for $30 \mathrm{~min}$. The obtained precipitates were filtered, washed with ethanol, and then dried.

Synthesis of $\mathrm{MAPbX}_{3} \mathrm{NCs} @ \mathrm{~Pb}-\mathrm{MOF}$ and $\mathrm{CsPbX}_{3} \mathrm{NCs} @ \mathrm{~Pb}-\mathrm{MOF}$ powder. The $\operatorname{MAX}(\mathrm{X}=\mathrm{I}, \mathrm{Br}, \mathrm{Cl})$ were synthesized according to our previous reports ${ }^{65}$. Briefly, $\mathrm{CH}_{3} \mathrm{NH}_{3} \mathrm{X}(\mathrm{X}=\mathrm{Cl}, \mathrm{Br}$, or I) was synthesized by the reaction of methylamine and $\mathrm{HBr}$ with the molar ratio of $1: 1$. After reaction for $2 \mathrm{~h}$, the precipitate was obtained by rotary evaporation, followed by washing with diethyl ether, and then dried under vacuum. For the synthesis of $\mathrm{MAPbX}_{3} \mathrm{NCs} @ \mathrm{~Pb}-\mathrm{MOF}$, the Pb-MOF $(200$ $\mathrm{mg}$ ) was dispersed in hexane $(10 \mathrm{~mL})$ with stirring at room temperature. For the typical synthesis of $\mathrm{MAPbBr}_{3} \mathrm{NCs@Pb-MOF,} \mathrm{the} \mathrm{Pb-MOF} \mathrm{(200} \mathrm{mg)} \mathrm{was} \mathrm{dispersed}$ in hexane $(10 \mathrm{~mL})$ with stirring at room temperature. Then $500 \mu \mathrm{L} \mathrm{MABr} / n$ butanol solution $(10 \mathrm{mg} / \mathrm{mL})$ was added to the $\mathrm{Pb}-\mathrm{MOF} /$ hexane suspension. After $30 \mathrm{~s}$, the colored powder was collected by filtration and rinsed with $n$-butanol then dried at $80^{\circ} \mathrm{C}$ for $5 \mathrm{~min}$ before further characterization. $\mathrm{MAPbBr}_{x} \mathrm{Cl}_{3-x} \mathrm{NCs} @ \mathrm{~Pb}-$ MOF and $\mathrm{MAPbBr}_{x} \mathrm{I}_{3-x}$ NCs NCs@Pb-MOF were fabricated via the similar strategy with the variation of the MAX/n-butanol solution constitution. The synthesis of $\mathrm{CsPbBr} \mathrm{Pr}_{3} \mathrm{NCs} @ \mathrm{~Pb}-\mathrm{MOF}$ was similar to the $\mathrm{MAPbBr}_{3} \mathrm{NCs} @ \mathrm{~Pb}-\mathrm{MOF}$ The difference was that the $\mathrm{MABr} / n$-butanol solution $(10 \mathrm{mg} / \mathrm{mL})$ and hexane were replaced with $\mathrm{CsBr} / \mathrm{methanol}$ solution $(20 \mathrm{mg} / \mathrm{mL})$ and toluene, respectively. For the quenching of $\mathrm{MAPbBr}_{3} \mathrm{NCs} @ \mathrm{~Pb}-\mathrm{MOF}$, the $\mathrm{MAPbBr}_{3} \mathrm{NCs} @ \mathrm{~Pb}-\mathrm{MOF}$ powder was dispersed in methanol with stirring at $25^{\circ} \mathrm{C}$. After $10 \mathrm{~min}$, the powder was collected by centrifugation and then dried at $80^{\circ} \mathrm{C}$.

Characterization. The powder XRD measurements were measured on a Bruker D8 Advance X-ray Diffractometer at $40 \mathrm{kV}$ and $40 \mathrm{~mA}$ using $\mathrm{Cu} K \alpha$ radiation $(\lambda=$ 
$1.5406 \AA$ ). The HR-TEM and scanning transmission electron microscope highangle annular dark-field (STEM HAADF) images were obtained from the FEI Talos F200X TEM instruments operated at an accelerating voltage of $200 \mathrm{kV}$. The SEM images were obtained from the JEOL JSM-7800F field emission scanning electron microscope (FESEM). The chemical compositions were determined by the XPS (Kratos Axis Ultra ${ }^{\mathrm{DLD}}$, all the binding energies were calibrated with the $\mathrm{C} 1 s$ peak at $284.8 \mathrm{eV}$ ) and X-Ray Fluorescence measurement (XRF-1800 spectrometer equipped with a Rh anode X-ray tube). FTIR spectra were measured by using a Nicolet 6700 spectrometer (USA). TGA was conducted on a Mettler Toledo analyzer from 30 to $700^{\circ} \mathrm{C}$ at a heating rate of $10^{\circ} \mathrm{C} / \mathrm{min}$ with an Ar flow rate of 50 $\mathrm{mL} / \mathrm{min}$. PL spectra were taken using a F-380 fluorescence spectrometer (Tianjin Gangdong Sci. \& Tech. Development Co., Ltd., China). The total luminescence spectra were characterized in the form of EEM (F-7000, Hitachi) with the scanning emission spectra varied from 450 to $600 \mathrm{~nm}$ by increasing the excitation wavelength from 300 to $550 \mathrm{~nm}$ at $5 \mathrm{~nm}$ increments. The PL decay curves were recorded on a PTI QM/TM/IM fluorescence spectrofluorometer. The absolute PLQY were detected using a fluorescence spectrometer with an integrated sphere executed at the $390 \mathrm{~nm}$ LED light source.

Security protection application. The $\mathrm{Pb}-\mathrm{MOF}$ ink solution was prepared by dissolving $2.15 \mathrm{~g} \mathrm{~Pb}\left(\mathrm{NO}_{3}\right)_{2}$ and $0.58 \mathrm{~g} 1,3,5-\mathrm{H}_{3} \mathrm{BTC}$ in $5 \mathrm{~mL}$ DMSO, then $11.25 \mathrm{~mL}$ ethanol (EtOH) and $7.5 \mathrm{~mL}$ ethylene glycol (EG) were added under vigorous stirring. Finally, the mixture was filtered through a $0.22 \mu \mathrm{m}$ syringe filter. The security printing tests were performed on a modified HP inkjet printer (HP Desk Jet 2132) with customized ink cartridges. The customized cartridge was washed with $\mathrm{H}_{2} \mathrm{O}$ and $\mathrm{EtOH}$ and dried in air. About $5 \mathrm{~mL}$ of $\mathrm{Pb}-\mathrm{MOF}$ ink solution was loaded into the empty cartridge and then loaded the cartridge into the printer. After printing, the substrate was dried in ambient, and then immersed into methanol solution for $30 \mathrm{~min}$ for a solvent development process to remote the residual solvents and dried again. For the decryption process, the printed substrate with $\mathrm{Pb}$-MOF patterns was loaded with $\mathrm{MABr} / n$-butanol $(10 \mathrm{mg} / \mathrm{mL})$ solution by a sprayer. After dried in ambient for several minutes, the shallow yellow green pattern can be observed by the naked eye. Under UV light illumination, the patterns showed bright green emission clearly. For the further encryption process, the substrate with patterns was immersed in methanol solution for about $10 \mathrm{~min}$, the green emission was gradually quenched and finally disappeared absolutely.

Data availability. All relevant data supporting the findings of this study are available from the corresponding authors on request.

Received: 24 April 2017 Accepted: 31 August 2017

Published online: 31 October 2017

\section{References}

1. Irie, M., Fukaminato, T., Sasaki, T., Tamai, N. \& Kawai, T. Organic chemistry: A digital fluorescent molecular photoswitch. Nature 420, 759-760 (2002).

2. Yoon, B. et al. Recent functional material based approaches to prevent and detect counterfeiting. J. Mater. Chem. C 1, 2388-2403 (2013).

3. Kishimura, A., Yamashita, T., Yamaguchi, K. \& Aida, T. Rewritable phosphorescent paper by the control of competing kinetic and thermodynamic self-assembling events. Nat. Mater. 4, 546-549 (2005).

4. Mutai, T., Satou, H. \& Araki, K. Reproducible on-off switching of solid-state luminescence by controlling molecular packing through heat-mode interconversion. Nat. Mater. 4, 685-687 (2005).

5. Sun, H. B. et al. Smart responsive phosphorescent materials for data recording and security protection. Nat. Commun. 5, 3601 (2014).

6. Luo, X. et al. Reversible switching of the emission of diphenyldibenzofulvenes by thermal and mechanical stimuli. Adv. Mater. 23, 3261-3262 (2011).

7. Medintz, I. L., Trammell, S. A., Mattoussi, H. \& Mauro, J. M. Reversible modulation of quantum dot photoluminescence using a protein-bound photochromic fluorescence resonance energy transfer acceptor. J. Am. Chem. Soc. 126, 30-31 (2004).

8. Qin, B. et al. Reversible photoswitchable fluorescence in thin films of inorganic nanoparticle and polyoxometalate assemblies. J. Am. Chem. Soc. 132, 2886-2888 (2010).

9. Jin., L. et al. Reversibly electroswitched quantum dot luminescence in aqueous solution. ACS Nano 5, 5249-5253 (2011).

10. Jiang, K. et al. Triple-mode emission of carbon dots: applications for advanced anti-counterfeiting. Angew Chem. Int. Ed. 55, 7231-7235 (2016).

11. Wang, J., Wang, C. -F. \& Chen, S. Amphiphilic egg-derived carbon dots: rapid plasma fabrication, pyrolysis process, and multicolor printing patterns. Angew Chem. Int. Ed. 124, 9431-9435 (2012).

12. Lou, Q. et al. Water-triggered luminescent "nano-bombs" based on supra(carbon nanodots). Adv. Mater. 27, 1389-1394 (2015).
13. Dong, $\mathrm{H}$. et al. Lanthanide nanoparticles: from design toward bioimaging and therapy. Chem. Rev. 115, 10725-10815 (2015).

14. $\mathrm{Li}, \mathrm{X}$. et al. A stimuli-responsive smart lanthanide nanocomposite for multidimensional optical recording and encryption. Angew Chem. Int. Ed. 129, $1-6$ (2017).

15. da Luz, L. et al. Inkjet printing of lanthanide-organic frameworks for anticounterfeiting applications. ACS Appl. Mater. Interfaces 7, (27115-27123 (2015).

16. Song, Z. et al. Invisible security ink based on water-soluble graphitic carbon nitride quantum dots. Angew Chem. Int. Ed. 128, 2823-2827 (2016).

17. Stranks, S. D. \& Snaith, H. J. Metal-halide perovskites for photovoltaic and light-emitting devices. Nat. Nanotechnol. 10, 391-402 (2015).

18. Zhou, $\mathrm{H}$. et al. Interface engineering of highly efficient perovskite solar cells. Science 345, 542-546 (2014).

19. Tan, Z. K. et al. Bright light-emitting diodes based on organometal halide perovskite. Nat. Nanotechnol. 9, 687-692 (2014).

20. Schmidt, L. C. et al. Nontemplate synthesis of $\mathrm{CH}_{3} \mathrm{NH}_{3} \mathrm{PbBr}_{3}$ perovskite nanoparticles. J. Am. Chem. Soc. 136, 850-853 (2014).

21. Zhang, F. et al. Brightly luminescent and color-tunable colloidal $\mathrm{CH}_{3} \mathrm{NH}_{3} \mathrm{PbX}_{3}$ $(\mathrm{X}=\mathrm{Br}, \mathrm{I}, \mathrm{Cl})$ quantum dots: potential alternatives for display technology. ACS Nano 9, 4533-4542 (2015).

22. Protesescu, L. et al. Nanocrystals of cesium lead halide perovskites $\left(\mathrm{CsPbX}_{3}\right.$, $\mathrm{X}=\mathrm{Cl}, \mathrm{Br}$, and I): novel optoelectronic materials showing bright emission with wide color gamut. Nano Lett. 15, 3692-3696 (2015).

23. Huang, H. et al. Colloidal lead halide perovskite nanocrystals: synthesis, optical properties and applications. NPG Asia Mater. 8, e328 (2016).

24. Akkerman, Q. A. et al. Tuning the optical properties of cesium lead halide perovskite nanocrystals by anion exchange reactions. J. Am. Chem. Soc. 137, 10276-10281 (2015).

25. Wang, Y. et al. Photon driven transformation of cesium lead halide perovskites from few-monolayer nanoplatelets to bulk phase. Adv. Mater. 28, 10637-10643 (2016).

26. James, S. L. Metal-organic frameworks. Chem. Soc. Rev. 32, 276-288 (2003).

27. Furukawa, H., Cordova, K. E., O'Keeffe, M. \& Yaghi, O. M. The chemistry and applications of metal-organic frameworks. Science 341, 1230444 (2013).

28. Li, W., Zhang, Y., Li, Q. \& Zhang, G. Metal-organic framework composite membranes: synthesis and separation applications. Chem. Eng. Sci. 135 , 232-257 (2015).

29. Li, W. et al. Transformation of metal-organic frameworks for molecular sieving membranes. Nat. Commun. 7, 11315 (2016).

30. Tian, H. \& Yang, S. Recent progresses on diarylethene based photochromic switches. Chem. Soc. Rev. 3, 85-97 (2004).

31. Sadeghzadeh, H. \& Morsali, A. Sonochemical synthesis and structural characterization of a nano-structure $\mathrm{Pb}$ (II) benzentricarboxylate coordination polymer: new precursor to pure phase nanoparticles of $\mathrm{Pb}(\mathrm{II})$ oxide. J. Coord Chem. 63, 713-720 (2010).

32. Kojima, A., Ikegami, M., Teshima, K. \& Miyasaka, T. Highly luminescent lead bromide perovskite nanoparticles synthesized with porous alumina media. Chem. Lett. 41, 397-399 (2012).

33. Longo, G., Pertegás, A., Martínez-Sarti, L., Sessolo, M. \& Bolink, H. J. Highly luminescent perovskite-aluminum oxide composites. J. Mater. Chem. C 3, 11286-11289 (2015).

34. Dirin, D. N. et al. Harnessing defect-tolerance at the nanoscale: highly luminescent lead halide perovskite nanocrystals in mesoporous silica matrixes. Nano Lett. 16, 5866-5874 (2016).

35. Malgras, V. et al. Observation of quantum confinement in monodisperse methylammonium lead halide perovskite nanocrystals embedded in mesoporous silica. J. Am. Chem. Soc. 138, 13874-13881 (2016).

36. Chen, Z., Gu, Z. -G., Fu, W. -Q., Wang, F. \& Zhang, J. A confined fabrication of perovskite quantum dots in oriented MOF thin film. ACS Appl. Mater. Interfaces 7, (28585-28742 (2015).

37. Wang, Y. et al. Ultrastable, highly luminescent organic-inorganic perovskitepolymer composite films. Adv. Mater. 28, 10710-10717 (2016).

38. Bowen Katari, J. E., Colvin, V. L. \& Alivisatos, A. P. X-ray photoelectron spectroscopy of CdSe nanocrystals with applications to studies of the nanocrystal surface. J. Phys. Chem. 98, 4109-4117 (1994).

39. Castner, D. G. X-ray Photoelectron spectroscopy sulfur $2 p$ study of organic thiol and disulfide binding interactions with gold surfaces. Langmuir 12, 5083-5086 (1996).

40. Cho, K., Han, S. -H. \& Suh, M. P. Copper-organic framework fabricated with $\mathrm{CuS}$ nanoparticles: synthesis, electrical conductivity, and electrocatalytic activities for oxygen reduction reaction. Angew Chem. Int. Ed. 55, 1-7 (2016).

41. Jiang, Z., Sun, H., Qin, Z., Jiao, X. \& Chen, D. Synthesis of novel ZnS nanocages utilizing ZIF-8 polyhedral template. Chem. Commun. 48, 3620-3622 (2012).

42. Esken, D. et al. ZnO@ZIF-8: stabilization of quantum confined $\mathrm{ZnO}$ nanoparticles by a zinc methylimidazolate framework and their surface structural characterization probed by $\mathrm{CO}_{2}$ adsorption. J. Mater. Chem. 21, 5907-5915 (2011). 
43. Tanaka, K. et al. Comparative study on the excitons in lead-halide-based perovskite type crystals $\mathrm{CH}_{3} \mathrm{NH}_{3} \mathrm{PbBr}_{3} \mathrm{CH}_{3} \mathrm{NH}_{3} \mathrm{PbI}_{3}$. Solid State Commun 127, 619-623 (2003).

44. Shekhah, O. et al. Controlling interpenetration in metal-organic frameworks by liquid-phase epitaxy. Nat. Mater. 8, 481-484 (2009).

45. Falcaro, P. R. et al. MOF positioning technology and device fabrication. Chem. Soc. Rev. 43, 5513-5560 (2014).

46. Lu, G., Farha, O. K., Zhang, W., Huo, F. \& Hupp, J. T. Engineering ZIF-8 thin films for hybrid MOF-based devices. Adv. Mater. 24, 3970-3974 (2012).

47. Hou, X. et al. Tunable solid-state fluorescent materials for supramolecular encryption. Nat. Commun. 6, 6884 (2015).

48. You, M. et al. Inkjet printing of upconversion nanoparticles for anti-counterfeit applications. Nanoscale 7, 4423-4431 (2015).

49. Singh, M., Haverinen, H. M., Dhagat, P. \& Jabbour, G. E. Inkjet printingprocess and its applications. Adv. Mater. 22, 673-685 (2010).

50. Wood, V. et al. Inkjet-printed quantum dot-polymer composites for full-color ac-driven displays. Adv. Mater. 21, 2151-2155 (2009).

51. Cui, L. et al. Fabrication of large-area patterned photonic crystals by ink-jet printing. J. Mater. Chem. 19, 5499-5502 (2009).

52. Zhuang, J. -L., Ar, D., Yu, X. -J., Liu, J. -X. \& Terfort, A. Patterned deposition of metal-organic frameworks onto plastic, paper, and textile substrates by inkjet printing of a precursor solution. Adv. Mater. 25, 4631-4635 (2013).

53. van de Hulst, H. C. Light Scattering by Small Particles (Wiley, New York, NY, USA, 1957)

54. Wolf, P. E. \& Maret, G. Weak localization and coherent backscattering of photons in disordered media. Phys. Rev. Lett. 55, 2696-2699 (1985).

55. Chen, D., Huang, F., Cheng, Y. B. \& Caruso, R. A. Mesoporous anatase $\mathrm{TiO}_{2}$ beads with high surface areas and controllable pore sizes: a superior candidate for high-performance dye-sensitized solar cells. Adv. Mater. 21, 2206-2210 (2009).

56. Ma., S., Liu, L., Bromberg, V. \& Singler, T. J. Fabrication of highly electrically conducting fine patterns via substrate-independent inkjet printing of musselinspired organic nano-material. J. Mater. Chem. C 2, 3885-3889 (2014).

57. Jeong, J. W. et al. High-resolution nanotransfer printing applicable to diverse surfaces via interface-targeted adhesion switching. Nat. Commun. 5, 5387 (2014).

58. $\mathrm{Gu}, \mathrm{Z}$. et al. Direct-writing multifunctional perovskite single crystal arrays by inkjet printing. Small 13, 1603217 (2017).

59. Bao, B. et al. Patterning fluorescent quantum dot nanocomposites by reactive inkjet printing. Small 11, 1649-1654 (2015).

60. Liang, K. et al. Biomimetic replication of microscopic metal-organic framework patterns using printed protein patterns. Adv. Mater. 27, 7293-7298 (2015)

61. Eames, C. et al. Ionic transport in hybrid lead iodide perovskite solar cells. Nat. Commun. 6, 7497 (2015).

62. Christians, J. A., Miranda Herrera, P. A. \& Kamat, P. V. Transformation of the excited state and photovoltaic efficiency of $\mathrm{CH}_{3} \mathrm{NH}_{3} \mathrm{PbI}_{3}$ perovskite upon controlled exposure to humidified air. J. Am. Chem. Soc. 137, 1530-1538 (2015).

63. Leguy, A. M. A. et al. Reversible hydration of $\mathrm{CH}_{3} \mathrm{NH}_{3} \mathrm{PbI}_{3}$ in films, single crystals, and solar cells. Chem. Mater. 27, 3397-3407 (2015)

64. Huang, W., Manser, J. S., Kamat, P. V. \& Ptasinska, S. Evolution of chemical composition, morphology, and photovoltaic efficiency of $\mathrm{CH}_{3} \mathrm{NH}_{3} \mathrm{PbI}_{3}$ perovskite under ambient conditions. Chem. Mater. 28, 303-311 (2015).

65. Huang, S. et al. Enhancing the stability of $\mathrm{CH}_{3} \mathrm{NH}_{3} \mathrm{PbBr}_{3}$ quantum dots by embedding in silica spheres derived from tetramethyl orthosilicate in "waterless" toluene. J. Am. Chem. Soc. 138, 5749-5752 (2016).

66. Glaser, T. et al. Infrared spectroscopic study of vibrational modes in methylammonium lead halide perovskites. J. Phys. Chem. Lett. 6, 2913-2918 (2015).
67. Babayigit, A., Ethirajan, A., Muller, M. \& Conings, B. Toxicity of organometal halide perovskite solar cells. Nat. Mater. 15, 247-251 (2016).

68. Hao, F., Stoumpos, C. C., Cao, D. H., Chang, R. P. H. \& Kanatzidis, M. G. Leadfree solid-state organic-inorganic halide perovskite solar cells. Nat. Photonics $\mathbf{8}$, 489-494 (2014).

69. Hong, W. -L. et al. Efficient low-temperature solution-processed lead-free perovskite infrared light-emitting diodes. Adv. Mater. 28, 8029-8036 (2016).

70. Jellicoe, T. C. et al. Synthesis and optical properties of lead-free cesium tin halide perovskite nanocrystals. J. Am. Chem. Soc. 138, 2941-2944 (2016).

71. Wang, A. et al. Controlled synthesis of lead-free and stable perovskite derivative $\mathrm{Cs}_{2} \mathrm{SnI}_{6}$ nanocrystals via a facile hot-injection process. Chem. Mater. 28, 8132-8140 (2016).

72. Leng, M. et al. Lead-free, blue emitting bismuth halide perovskite quantum dots. Angew Chem. Int. Ed. 55, 15012-15016 (2016).

\section{Acknowledgements}

This work is supported by the National Natural Science Foundation of China (21271179, 21607101) and the Program for New Century Excellent Talents (NCET-13-0364).

\section{Author contributions}

L.L. and C.Z. conceived the original research idea. Experiments, including Pb-MOF fabrication, $\mathrm{MAPbBr}_{3} \mathrm{NCs@Pb-MOF}$ synthesis, and inkjet-printing test were performed by C.Z., B.W., and W. L. C.Z., S.H., L.K., and Z.L. carried out characterizations and analyses including transmission electron microscope, scanning electron microscopy, powder diffraction, X-ray photoelectron spectroscopy, UV-vis absorption, PL, PL lifetime, and PLQY. The manuscript was co-written by L.L. and C.Z. All authors contribute to the discussion and revising of this manuscript.

\section{Additional information}

Supplementary Information accompanies this paper at https://doi.org/10.1038/s41467017-01248-2.

Competing interests: The authors declare no competing financial interests.

Reprints and permission information is available online at http://npg.nature.com/ reprintsandpermissions/

Publisher's note: Springer Nature remains neutral with regard to jurisdictional claims in published maps and institutional affiliations.

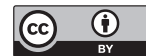

Open Access This article is licensed under a Creative Commons Attribution 4.0 International License, which permits use, sharing, adaptation, distribution and reproduction in any medium or format, as long as you give appropriate credit to the original author(s) and the source, provide a link to the Creative Commons license, and indicate if changes were made. The images or other third party material in this article are included in the article's Creative Commons license, unless indicated otherwise in a credit line to the material. If material is not included in the article's Creative Commons license and your intended use is not permitted by statutory regulation or exceeds the permitted use, you will need to obtain permission directly from the copyright holder. To view a copy of this license, visit http://creativecommons.org/ licenses/by/4.0/

(C) The Author(s) 2017 